\title{
Psychometric properties of morning joint stiffness duration and severity measures in patients with moderately to severely active rheumatoid arthritis
}

\author{
Elizabeth D. Bacci ${ }^{*}$, Amy M. DeLozier ${ }^{2}$, Chen-Yen Lin², Carol L. Gaich², Terence Rooney², Richard Hoffman ${ }^{2}$
} and Kathleen W. Wyrwich ${ }^{3}$

\begin{abstract}
Background: To assess the measurement properties of two single-item patient-reported outcome (PRO) measures that assessed the length of time (in minutes) and severity of morning joint stiffness (MJS) experienced each day.

Methods: Data from two Phase 3, randomized placebo-controlled (and active-controlled [RA-BEAM]), clinical studies assessing the safety and efficacy of baricitinib in adults with moderately to severely active rheumatoid arthritis (RA) were used to evaluate the psychometric properties of the Duration of MJS and Severity of MJS PROs.

Results: Test-retest reliability of Duration of MJS and Severity of MJS was supported through large intraclass correlation coefficients among stable patients (coefficient range for both studies: 0.88 to 0.93). In support of construct validity, moderate correlations were evidenced between Duration of MJS and other related patient- and clinician-reported assessments of RA symptoms and patient functioning, whereas moderate-to-strong correlations were evidenced between these same patient- and clinician-reported assessments and Severity of MJS. Statistically significant differences between the median and mean values of Duration of MJS and Severity of MJS for differing categories of RA disease severity supported known-groups validity. Finally, large and statistically significant differences in change scores from Day 1 to Week 12 for patients defined as responders versus non-responders using the American College of Rheumatology 20 criteria supported the responsiveness of both PROs.
\end{abstract}

Conclusion: Duration of MJS and Severity of MJS PROs demonstrated reliability, validity, and responsiveness in adults with moderately to severely active RA, supporting the measurement of these key symptoms in clinical trials.

Keywords: Rheumatoid arthritis, Morning joint stiffness, Patient-reported outcome, Psychometric, Reliability, Validity, Responsiveness

\section{Background}

Rheumatoid arthritis (RA) is a systemic, inflammatory, autoimmune disease. The expression and outcomes of the disease vary, ranging from mild, limited disease to severe disease that is associated with progressive joint destruction, significantly compromised health-related quality of life (HRQOL), and reduced survival. Some clinical symptoms of RA, such as morning joint stiffness (MJS), may follow a circadian pattern and the rhythm of

\footnotetext{
* Correspondence: elizabeth.bacci@evidera.com

${ }^{1}$ Evidera, Inc, 1417 4th Avenue, Suite 510, Seattle, WA 98101, USA

Full list of author information is available at the end of the article
}

pro-inflammatory cytokines, such as interleukin-6 (IL-6). The increase in nocturnal anti-inflammatory cortisol seen in patients with RA is generally insufficient to suppress the ongoing joint inflammation, often resulting in joint stiffness in the morning [1-3].

Prior research illustrates the importance of early morning stiffness or difficulty moving joints for patients with RA. In a study of 916 patients with recent onset of RA (disease duration $\leq 24$ months), Westhoff et al. [4] reported that for many patients, morning stiffness impacts their HRQOL, functional capability, and ability to continue to work. These findings are supported by 
recent survey research conducted by Mattila et al. [5] in patients with RA (duration $>6$ months) who experience impairment of morning function at least 3 times per week. Of the 534 working respondents, $47 \%$ indicated morning stiffness affected their work performance, and $33 \%$ indicated it led to a late arrival at work. Of the 224 retired participants, 159 (71\%) stopped working earlier than their expected retirement age, with $64 \%$ of these participants giving RA-related morning stiffness as a reason for their retirement [5]. Indeed, MJS is an important and clinically meaningful element of disease activity [6].

Patient-reported outcome (PRO) measures of symptoms like MJS are important tools to aid clinicians in treating patients with RA, facilitate doctor-patient communication to improve the quality of patient care, and contribute to better patient outcomes [7]. Despite the importance of MJS to patients with RA [4, 6], the assessment of MJS is not currently a measured component in several recommended endpoints in clinical trials, such as the American College of Rheumatology (ACR), Disease Activity Score modified to include the 28 diarthrodial joint count (DAS28), Clinical Disease Activity Index (CDAI), or Simplified Disease Activity Index (SDAI).

To address this need, two daily electronic PRO diary items were created to assess both the duration and severity of MJS from the patient's perspective. The content validity of these two items were supported through a targeted literature review, interviews with health-care providers, and qualitative concept elicitation and cognitive debriefing interviews with patients with RA [8]. These interviews confirmed the relevance of duration and severity of MJS as symptoms of RA, as well as the appropriateness of terminology used to assess these symptoms.

This study reports the assessment of the psychometric properties (i.e., reliability, validity, and responsiveness) of two PRO items administered daily, the Duration of MJS and Severity of MJS, in patients with moderately to severely active RA who participated in two Phase 3 clinical trials for baricitinib RA-BEAM and RA-BUILD.

\section{Methods \\ Patient population \\ RA-BEAM}

RA-BEAM $(N=1305)$ was a randomized, double-blind, double-dummy, placebo- and active-controlled, parallelarm, 52-week study designed to assess improvements in disease activity, structural preservation, and PROs including physical function, safety, and tolerability with oral baricitinib 4-mg once daily in patients with RA who had inadequate responses to methotrexate. Full details regarding the primary efficacy and safety outcomes of this study have been reported previously [9]. Briefly, patients were aged $\geq 18$ years with active RA ( $\geq 6 / 68$ tender and $\geq 6 / 66$ swollen joints; serum high-sensitivity C-reactive protein $[\mathrm{hsCRP}] \geq 6 \mathrm{mg} / \mathrm{L}$ ). Comparisons were made to placebo and to adalimumab, a tumor necrosis factor (TNF)- $\alpha$ inhibitor and a standard-of-care biologic disease-modifying antirheumatic drug (DMARD) in this setting.

\section{RA-BUILD}

RA-BUILD $(N=684)$ was a randomized, double-blind, placebo-controlled, parallel-group 24-week study designed to assess improvements in disease activity, structural preservation, and PROs including physical function, safety, and, tolerability with oral baricitinib 2$\mathrm{mg}$ and 4-mg once daily in patients with RA who were refractory to or intolerant of conventional synthetic DMARDs (csDMARDs). Full details regarding the primary efficacy and safety outcomes of this study have been reported previously [10]. Briefly, patients were aged $\geq 18$ years with active rheumatoid arthritis ( $\geq 6 / 68$ tender and $\geq 6 / 66$ swollen joints; hsCRP $\geq 3.6 \mathrm{mg} / \mathrm{L}$ [upper limit of normal $3.0 \mathrm{mg} / \mathrm{L}]$ ) and an insufficient response (despite prior therapy) or intolerance to $\geq 1$ csDMARDs. Comparisons were made to placebo.

For both studies, visits occurred on Weeks $0,1,2,4,8$, $12,14,16,20$, and 24 , and for RA-BEAM, the visits continued to Weeks 28, 32, 40, and 52. Both studies were conducted with informed consent, under institutional review board approval, and in accordance with the Declaration of Helsinki (ClinicalTrials.gov number NCT01710358 [RABEAM] and NCT01721057 [RA-BUILD]).

\section{Instruments used in the psychometric analyses Patient-reported outcomes (PROs)}

Duration of morning joint stiffness, severity of morning joint stiffness, severity of worst tiredness, and severity of worst joint pain Duration of MJS is a single-item PRO designed to capture information on self-reported length of time, in minutes, that a patient's MJS lasted each day. Specifically, patients were asked: "Please indicate how long your morning joint stiffness lasted today," and responded with the number of hours and minutes. Durations recorded as $>12 \mathrm{~h}(720 \mathrm{~min})$ were censored at $720 \mathrm{~min}$.

Severity of MJS, Severity of Worst Tiredness, and Severity of Worst Joint Pain are all single-item PROs designed to capture the severity of MJS, worst tiredness, and worst joint pain experienced that day, respectively. Patient's were asked to complete each at the end of their day. Specifically, for Severity of MJS, patients were asked: "Please rate the overall level of morning joint stiffness you had from the time you woke up today." All three of these PROs are anchored at 0 and 10, where 0 represents "no joint stiffness," "no tiredness," or "no joint pain," and 10 represents "joint stiffness as bad as 
you can imagine," "tiredness as bad as you can imagine," or "joint pain as bad as you can imagine," respectively.

For RA-BEAM and RA-BUILD, all four PROs were assessed using an electronic diary on a daily basis through Week 12. The Day 1 assessment was the first assessment at the end of the patient's day following the randomization visit (Week 0 , Visit 2). The Week 1 assessment refers to the weekly average values from Days 2 to 8 . Assessments at Weeks 2, 4, 8, and 12 refer to weekly average values of the 7 days prior to Weeks 2,4 , 8 , and 12 visits, respectively. Recognizing that late-shift workers, individuals who work outside of the hours of 9 am until 5 pm, could not complete the electronic diary (at home) at the end of Day 1, the Day 2 assessment (if available) was used to impute missing Day 1 values so that more patients could be included in the psychometric analyses utilizing the Day 1 data.

Medical Outcomes Study 36-item Short Form Health Survey version 2 Acute (SF-36) The SF-36 is a generic, 36-item PRO that measures general health status. The SF-36 includes eight domains of health status evaluated over the previous week: physical function, role limitations-physical, bodily pain, general health perceptions, vitality, social function, role limitations-emotional, and mental health. Two component scores, the Physical Component Score (PCS) and the Mental Component Score (MCS), are derived based on the 8 domain scores [11]. Domain and component scores are derived using established formulas [11], with higher scores indicating better health status or functioning. Acceptable psychometric properties of this instrument have been demonstrated elsewhere [12, 13].

Health Assessment Questionnaire-Disability Index (HAQ-DI) The HAQ-DI assesses patients' physical function or disability over the past week. The HAQ-DI contains 24 questions that query the degree of difficulty a person has in accomplishing tasks in 8 functional areas (dressing, arising, eating, walking, hygiene, reaching, gripping, and activities). Responses in each functional area are scored from 0 , indicating "no difficulty," to 3, indicating "inability to perform a task" in that area. The HAQ-DI total score, ranging from 0 to 3 (higher values indicate worse functioning), is obtained by summing the highest score within each functional area and dividing by the number of functional areas answered [14]. The reliability and validity of this instrument have been documented previously [15].

Quick Inventory of Depressive Symptomatology SelfRated-16 (QIDS-SR 16) The QIDS-SR $_{16}$ is a 16-item PRO intended to assess the existence and severity of symptoms of depression as listed in the American
Psychiatric Association's Diagnostic and Statistical Manual of Mental Disorders, 4th Edition (DSM-IV) [16]. Patients were asked to consider each statement as it relates to the way they have felt for the past 7 days. There is a unique 4-point ordinal scale for each item, with scores ranging from 0 to 3 reflecting increasing depressive symptoms as the item score increases. The instrument measures 9 core symptom domains that are used to define a depressive episode: sad mood; concentration; self criticism; suicidal ideation; interest; energy/fatigue; sleep disturbance; decrease or increase in appetite or weight; and psychomotor agitation or retardation. The QIDS-SR 16 total score is derived as the sum of the scores across the 9 scale domains. The psychometric properties, including reliability, validity, and sensitivity to change of this instrument have been demonstrated elsewhere [17].

Patient's assessment of pain Patient's current pain was assessed at each study visit with the use of a 0- to 100$\mathrm{mm}$ visual analogue scale (VAS), with higher scores indicating more severe pain.

Patient's Global Assessment of Disease Activity (PtGA) The PtGA, assessed as their current disease activity, was assessed at each study visit and is recorded on a 0 - to 100-mm VAS, with higher scores indicating more active RA.

\section{Clinician-reported assessments}

Physician's Global Assessment of Disease Activity (PhGA) The PhGA was assessed at each study visit and is recorded on a 0-to 100-mm VAS, with higher scores indicating or more active RA.

\section{Clinical signs and symptoms measures}

American College of Rheumatology 20 (ACR20) An ACR20 response (i.e., a binary variable indicating achieving or not achieving a response) was measured at each study visit and is defined as at least a $20 \%$ improvement from baseline in both tender joint count (TJC) (0 to 68) and swollen joint count (SJC) (0 to 66), and in at least 3 of the following 5 assessments: patient's assessment of pain, PtGA, PhGA, HAQ-DI, and high-sensitivity Creactive protein.

Clinical Disease Activity Index (CDAI) The CDAI is a tool for measurement of disease activity in RA that integrates measures of physical examination, patient selfassessment, and evaluator assessment [18]. The CDAI was assessed at each study visit and is calculated by adding together scores from the following assessments: 
number of swollen joints (0 to 28), number of tender joints (0 to 28), PtGA on a VAS (0 to $10 \mathrm{~cm}$ ), PhGA on a VAS $(0$ to $10 \mathrm{~cm})$. Total scores are calculated using established formulas [18]. Thresholds have been established for the CDAI (remission: 0.0 to $\leq 2.8$; low disease activity: $>2.8$ to $\leq 10$; moderate disease activity: $>10$ to $\leq 22$; high disease activity: $>22$ to $\leq 76)$ [19].

Disease Activity Score (28 joints) (DAS28) The DAS28 is a composite score that is based on a 28-joint count (both TJC 0 to 28 and SJC 0 to 28), hsCRP or erythrocyte sedimentation rate (ESR), and PtGA and was measured at each study visit. Total scores are calculated using established formulas [20]. Patients can be categorized into 4 groups (remission: <2.6; low disease activity: $\geq 2.6$ to $\leq 3.2$; moderate disease activity: $>3.2$ to $\leq 5.1$; high disease activity: $>5.1$ ).

\section{Statistical analyses}

The distribution of scores for the Duration of MJS and Severity of MJS PROs was assessed using descriptive statistics at Day 1, including mean (SD), median, range, and ceiling/floor effects.

\section{Reliability (test-retest)}

Test-retest reliability was assessed in stable patients during the interval between Week 1 and 2 and again between Week 4 and 8 . Stable patients were defined as those with $\leq 5$ point difference [21] on the 0 to 100 PtGA between each assessment period. Intraclass correlation coefficients (ICCs) were calculated between the initial (Week 1 or 4 ) and retest (Week 2 or 8 ) scores to evaluate test-retest reliability. An ICC of $\geq 0.70$ was considered good agreement [22].

\section{Convergent and discriminant validity (construct validity)}

Construct validity was assessed by Pearson correlations at Day 1 and Week 12 between the scores of Duration of MJS and Severity of MJS, and the scores of other clinical/PRO endpoints: Severity of Worst Tiredness, Severity of Worst Joint Pain, SF-36 domain and component scores, HAQ-DI, QIDS-SR 16 , patient's assessment of pain, PtGA, TJC28, SJC28, PhGA, and hsCRP. Cohen's conventions were used to interpret the absolute value of the correlation results, where a correlation $>0.5$ is large, 0.3 to 0.5 is moderate, 0.1 to $<0.3$ is small, and $<0.1$ is insubstantial [23].

It was hypothesized that moderate or large correlations supporting convergent validity would be demonstrated between Duration of MJS and Severity of MJS and the PRO instruments measuring concepts related to RA symptoms (SF-36 PCS, SF-36 Bodily Pain), their impact on functioning (SF-36 Social Functioning, SF-36 Vitality, SF-36 Physical Functioning, HAQ-DI), and clinician-reported/laboratory assessments of disease activity (TJC28, SJC28, PhGA, and hsCRP). Discriminant validity was assessed by Pearson correlations at Day 1 and at Week 12 between Duration of MJS, Severity of MJS, and PROs measuring distally related concepts (SF36 MCS, SF-36 Role Emotional, QIDS-SR ${ }_{16}$ ) where small correlations were hypothesized.

\section{Known-groups validity}

Known-groups validity was evaluated using the KruskalWallis test to distinguish median Duration of MJS and an analysis of variance (ANOVA) model to distinguish mean Severity of MJS between subgroups defined by the DAS28-ESR thresholds $(<2.6 ; \geq 2.6$ and $\leq 3.2 ;>3.2$ and $\leq 5.1$; $>5.1)$ measured at Day 1 and Week 4 , and CDAI ( 0.0 to $\leq 2.8 ;>2.8$ to $\leq 10 ;>10$ to $\leq 22$; and $>22$ to $\leq 76$ ) measured at Day 1 and Week 4. The Scheffé adjustment was used for multiple comparisons. Subgroups were combined in instances of small sample sizes (i.e., $<5 \%$ of the total sample size for the subgroup).

\section{Responsiveness}

Due to anticipated skewness of Duration of MJS PRO, responsiveness was evaluated using a nonparametric randomization-based analysis of covariance (ANCOVA) methodology [24] to assess significant differences in median change in Duration of MJS from Day 1 to Week 12 between ACR20 responders and nonresponders at Week 12, controlling for Duration of MJS at Day 1. Responsiveness was evaluated using an ANCOVA methodology to assess significant differences in mean change in Severity of MJS. Responsiveness was also assessed using disease activity as measured by DAS28-hsCRP at Week 12, using the following subgroups: DAS28-hsCRP $<2.6$, DAS28-hsCRP $\geq 2.6$ and DAS28-hsCRP $\leq 3.2$, and DAS28-hsCRP $>3.2$. An overall statistically significant difference $(p<0.05)$ with statistically significant subgroup comparisons was hypothesized.

$\mathrm{SAS}^{\circ}$ statistical software Version 9,4 (SAS Institute Inc., Cary, NC, USA) was used to conduct all analyses.

\section{Results}

Baseline demographics for the total modified intent-totreat population, patients with Day 1 diary scores, and patients with Week 12 diary scores are provided in Table 1. Baseline and Week 12 scores for Duration of MJS PRO, Severity of MJS PRO, and other assessments are presented in Table 2, while score distributions for Duration of MJS PRO and Severity of MJS PRO are found in Additional file 1: Table S1. As can be seen in Tables 1 and 2, there was a large amount of missing data at the Day 1 assessment period. This missing data was due to multiple reasons as shown in Additional file 1: Table S2, such as the diary device alarm not sounding until the following day or the diary device 


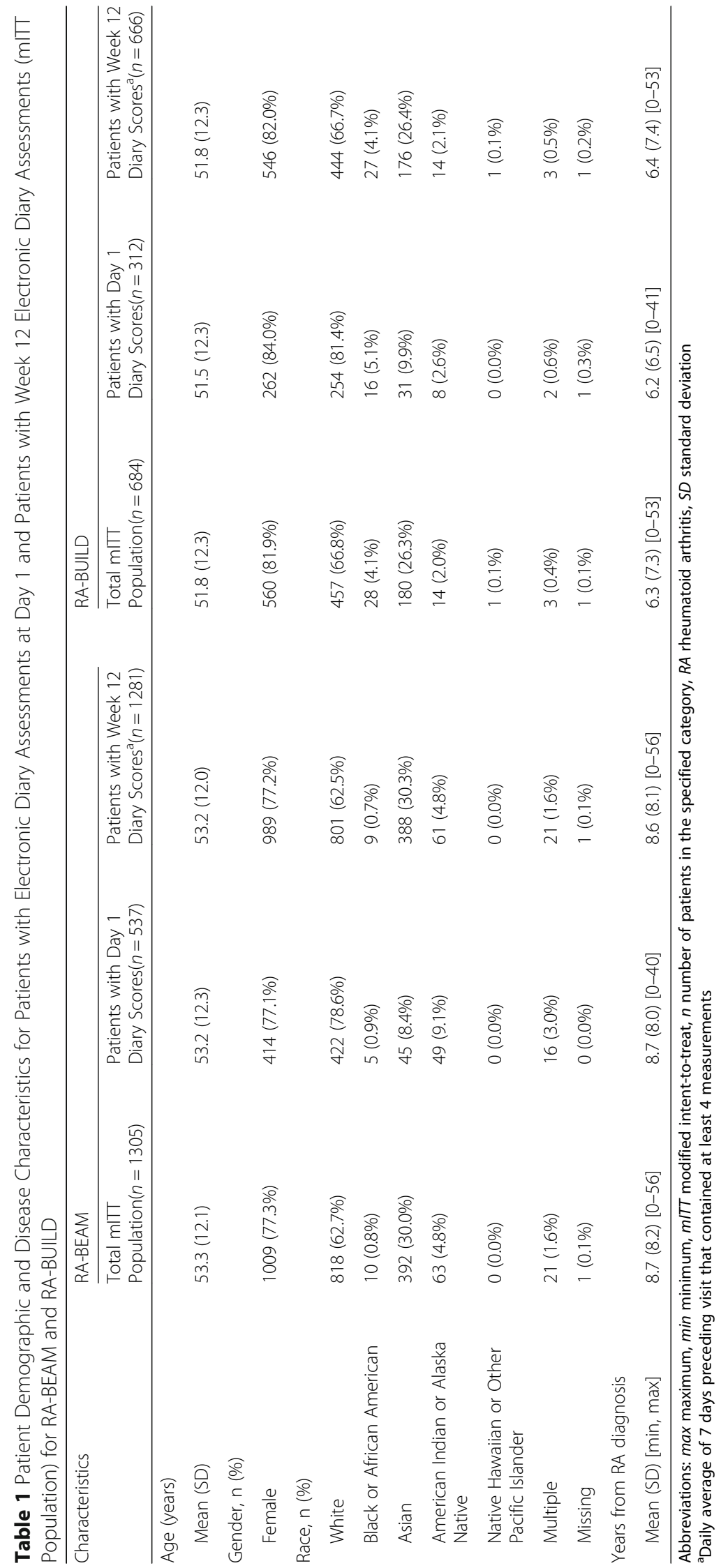


Table 2 Instrument Scores at Day 1 and Week 12 for RA-BEAM and RA-BUILD

\begin{tabular}{|c|c|c|c|c|}
\hline \multirow[t]{2}{*}{ Instrument } & \multicolumn{2}{|l|}{ RA-BEAM $^{a}$} & \multicolumn{2}{|l|}{ RA-BUILD ${ }^{b}$} \\
\hline & Day 1 & Week 12 & Day 1 & Week 12 \\
\hline Duration of MJS, Mean (SD) & $152.8(180.8)$ & $96.7(144.7)$ & $160.7(174.8)$ & $103.5(147.5)$ \\
\hline Severity of MJS, Mean (SD) & $5.8(2.2)$ & $3.5(2.4)$ & $5.7(2.1)$ & $3.7(2.4)$ \\
\hline Severity of Worst Tiredness, Mean (SD) & $5.8(2.1)$ & $4.0(2.3)$ & $6.0(2.1)$ & $4.1(2.4)$ \\
\hline Severity of Worst Joint Pain, Mean (SD) & $6.1(2.0)$ & $4.0(2.3)$ & $6.0(2.1)$ & $4.2(2.4)$ \\
\hline Patient's Assessment of Pain, Mean (SD) & $60.8(22.3)$ & $34.9(24.1)$ & $58.0(22.1)$ & $36.5(24.9)$ \\
\hline $\begin{array}{l}\text { Patient's Global Assessment of Disease } \\
\text { Activity, Mean (SD) }\end{array}$ & $62.4(21.8)$ & $36.3(23.5)$ & $60.7(21.1)$ & $37.7(24.0)$ \\
\hline \multicolumn{5}{|l|}{ SF-36 } \\
\hline Role Physical Domain, Mean (SD) & $35.5(10.4)$ & $41.8(10.2)$ & $35.3(9.2)$ & $40.7(9.6)$ \\
\hline Bodily Pain Domain, Mean (SD) & $34.7(7.8)$ & $42.5(8.7)$ & $34.9(7.6)$ & $41.9(9.0)$ \\
\hline General Health Domain, Mean (SD) & $36.8(8.5)$ & $41.6(8.9)$ & $36.8(8.3)$ & $42.0(9.4)$ \\
\hline Social Functioning Domain, Mean (SD) & $40.8(11.6)$ & $45.4(10.5)$ & $40.2(11.2)$ & $44.7(10.3)$ \\
\hline Vitality Domain, Mean (SD) & $43.7(10.0)$ & $49.4(9.8)$ & $41.9(10.1)$ & $48.4(10.0)$ \\
\hline Role Emotional Domain, Mean (SD) & $41.1(12.6)$ & $45.5(10.9)$ & $40.9(12.3)$ & $44.8(11.2)$ \\
\hline Mental Health Domain, Mean (SD) & $43.0(11.3)$ & $47.1(10.7)$ & $42.9(11.6)$ & $47.1(11.3)$ \\
\hline Physical Functioning Domain, Mean (SD) & $32.2(10.4)$ & $38.7(10.9)$ & $32.2(10.2)$ & $38.2(10.9)$ \\
\hline Mental Component Score, Mean (SD) & $46.4(11.8)$ & $49.8(10.8)$ & $45.7(11.8)$ & $49.3(11.1)$ \\
\hline Physical Component Score, Mean (SD) & $32.3(8.6)$ & $39.4(9.3)$ & $32.3(8.3)$ & $38.8(9.4)$ \\
\hline QIDS-SR 16 Total Score, Mean (SD) & $8.0(5.0)$ & $5.9(4.2)$ & $8.4(4.9)$ & $6.2(4.3)$ \\
\hline HAQ-DI Total Score, Mean (SD) & $1.56(0.68)$ & $1.03(0.70)$ & $1.52(0.61)$ & $1.03(0.66)$ \\
\hline PhGA, Mean (SD) & $65.0(16.9)$ & $31.0(21.7)$ & $63.5(17.4)$ & $33.9(22.4)$ \\
\hline CDAl, Mean (SD) & $37.83(12.55)$ & $18.68(13.54)$ & $36.05(12.12)$ & $18.04(13.15)$ \\
\hline DAS28-ESR, Mean (SD) & $6.43(0.97)$ & $4.62(1.46)$ & $6.22(0.97)$ & $4.50(1.37)$ \\
\hline DAS28-hsCRP, Mean (SD) & $5.73(0.94)$ & $3.93(1.39)$ & $5.55(0.91)$ & $3.84(1.35)$ \\
\hline
\end{tabular}

Abbreviations: CDAI Clinical Disease Activity Index, DAS28 Disease Activity Score modified to include the 28 diarthrodial joint count, ESR erythrocyte sedimentation rate, HAQ-DI Health Assessment Questionnaire-Disability Index, $h s C R P$ high-sensitivity C-reactive protein, $n$ number of patients, MJS morning joint stiffness, PhGA Physician's Global Assessment of Disease Activity, QIDS-SR ${ }_{16}$ Quick Inventory of Depressive Symptomatology Self-Rated-16, SD standard deviation, SF-36, 36-Item Short Form Health Survey version 2 with Acute recall

${ }^{a}$ Daily diary responses were $n=537$ (Day 1) and $n=1281$ (Week 12); nondiary assessments had approximately $n=1301$ (Day 1 ) and $n=1250$ (Week 12)

${ }^{b}$ Daily diary responses were $n=310$ (Day 1) and $n=666$ (Week 12); nondairy assessments had approximately $n=680$ (Day 1 ) and $n=644$ (Week 12)

being given to the patient after Day 1 . Sensitivity analyses with the imputation for missing data at Day $1(n=1041$ for RA-BEAM and $n=563$ for RA-BUILD, respectively) demonstrated similar comparisons to the results presented in this document.

\section{Reliability (test-retest)}

For RA-BEAM and RA-BUILD, the ICCs for weekly mean Duration of MJS and Severity of MJS ranged from 0.88 to 0.91 from Week 1 to 2 (RA-BEAM $n=412$; RA-BUILD $n=185$ ) and from 0.90 to 0.93 from Week 4 to Week 8 (RA-BEAM $n=417$; RA-BUILD $n=215$ ). These values provide evidence for substantial agreement between assessment periods among stable patients.

\section{Convergent and discriminant validity}

Results supporting convergent validity of Duration of MJS and Severity of MJS are found in Table 3. For
Duration of MJS, small-to-moderate associations were found between Duration of MJS and other assessments of RA symptoms, assessments of function, and clinicianreported assessments at Day 1 and Week 12. At Day 1 and Week 12, respectively, Duration of MJS was most strongly associated with Severity of MJS (RA-BEAM $r$ $=0.36$ and 0.45 ; RA-BUILD $r=0.35$ and 0.52 ) and Severity of Worst Joint Pain (RA-BEAM $r=0.29$ and 0.41; RA-BUILD $r=0.30$ and 0.47), followed by the patient's assessment of pain (RA-BEAM $r=0.24$ and 0.38; RA-BUILD $r=0.23$ and 0.39) and PtGA (RABEAM $r=0.19$ and 0.36; RA_BUILD $r=0.28$ and 0.37).

For Severity of MJS, moderate-to-large associations were found between Severity of MJS and other assessments of RA symptoms, assessments of function, and clinician-reported assessments at Day 1 and Week 12. At Day 1 and Week 12, respectively, Severity of MJS was most strongly associated with Severity of Worst Joint 
Table 3 Pearson Correlations between Duration of MJS, Severity of MJS, Severity of Worst Tiredness, and Other Instruments in RABEAM and RA-BUILD

\begin{tabular}{|c|c|c|c|c|c|c|c|c|}
\hline \multirow[t]{3}{*}{ Instruments } & \multicolumn{4}{|c|}{ Duration of MJS } & \multicolumn{4}{|c|}{ Severity of MJS } \\
\hline & \multicolumn{2}{|c|}{ RA-BEAM } & \multicolumn{2}{|l|}{ RA-BUILD } & \multicolumn{2}{|c|}{ RA-BEAM } & \multicolumn{2}{|l|}{ RA-BUILD } \\
\hline & Number & $r$ & Number & $r$ & Number & $r$ & Number & $r$ \\
\hline \multicolumn{9}{|l|}{ Day 1} \\
\hline Duration of MJS & - & - & - & - & 537 & $0.36^{* * *}$ & 311 & $0.35^{* * *}$ \\
\hline Severity of MJS & 537 & $0.36^{* * *}$ & 311 & $0.35^{* * *}$ & - & - & - & - \\
\hline Severity of Worst Tiredness & 537 & $0.21^{* * *}$ & 311 & 0.09 & 537 & $0.66^{* * *}$ & 311 & $0.52^{* * *}$ \\
\hline Severity of Worst Joint Pain & 537 & $0.29 * * *$ & 310 & $0.30^{* * *}$ & 537 & $0.79 * * *$ & 310 & $0.75^{* * *}$ \\
\hline \multicolumn{9}{|l|}{ SF-36 } \\
\hline Role Physical Domain & 535 & $-0.24^{* * *}$ & 311 & $-0.23^{* * *}$ & 535 & $-0.43^{* * *}$ & 312 & $-0.40^{* * *}$ \\
\hline Bodily Pain Domain & 535 & $-0.30^{* * *}$ & 311 & $-0.29 * * *$ & 535 & $-0.65^{* * *}$ & 312 & $-0.51^{* * *}$ \\
\hline General Health Domain & 535 & $-0.09^{*}$ & 311 & $-0.18^{* *}$ & 535 & $-0.30^{* * *}$ & 312 & $-0.25^{* * *}$ \\
\hline Social Functioning Domain & 535 & $-0.16^{* * *}$ & 311 & $-0.24^{* * *}$ & 535 & $-0.38^{* * *}$ & 312 & $-0.41^{* * *}$ \\
\hline Vitality Domain & 535 & $-0.21^{* * *}$ & 311 & $-0.18^{* *}$ & 535 & $-0.42^{* * *}$ & 312 & $-0.32^{* * *}$ \\
\hline Role Emotional Domain & 535 & -0.07 & 311 & $-0.12^{*}$ & 535 & $-0.27^{* * *}$ & 312 & $-0.25^{* * *}$ \\
\hline Mental Health Domain & 535 & $-0.10^{*}$ & 311 & $-0.20^{* * *}$ & 535 & $-0.31^{* * *}$ & 312 & $-0.27^{* * *}$ \\
\hline Physical Functioning Domain & 535 & $-0.25^{* * *}$ & 311 & $-0.28^{* * *}$ & 535 & $-0.51 * * *$ & 312 & $-0.47^{* * *}$ \\
\hline Mental Component Score & 535 & -0.07 & 311 & $-0.15^{* *}$ & 535 & $-0.26^{* * *}$ & 312 & $-0.25^{* * *}$ \\
\hline Physical Component Score & 535 & $-0.29 * * *$ & 311 & $-0.27^{* * *}$ & 535 & $-0.54^{* * *}$ & 312 & $-0.44^{* * *}$ \\
\hline HAQ-DI Total Score & 535 & $0.22^{* * *}$ & 311 & $0.26^{* * *}$ & 535 & $0.53^{* * *}$ & 312 & $0.46^{* * *}$ \\
\hline QIDS-SR 16 Total Score & 535 & $0.15^{* * *}$ & 311 & $0.26^{* * *}$ & 535 & $0.34^{* * *}$ & 312 & $0.28^{* * *}$ \\
\hline Patient's Assessment of Pain & 535 & $0.24^{* * *}$ & 311 & $0.23^{* * *}$ & 535 & $0.59 * * *$ & 312 & $0.54^{* * *}$ \\
\hline PtGA & 535 & $0.19^{* * *}$ & 311 & $0.28^{* * *}$ & 535 & $0.59^{* * *}$ & 312 & $0.53^{* * *}$ \\
\hline Tender Joint Count 28 & 537 & $0.09^{*}$ & 311 & $0.15^{* *}$ & 537 & $0.26^{* * *}$ & 312 & $0.43^{* * *}$ \\
\hline Swollen Joint Count 28 & 537 & 0.06 & 311 & $0.13^{*}$ & 537 & $0.21^{* * *}$ & 312 & $0.24^{* * *}$ \\
\hline PhGA & 535 & $0.19^{* * *}$ & 304 & $0.19^{* * *}$ & 535 & $0.32^{* * *}$ & 305 & $0.33^{* * *}$ \\
\hline hsCRP & 535 & $0.20^{* * *}$ & 311 & $0.27^{* * *}$ & 535 & $0.48^{* * *}$ & 312 & $0.53^{* * *}$ \\
\hline \multicolumn{9}{|l|}{ Week $12^{\mathrm{a}}$} \\
\hline Duration of MJS & - & - & - & - & 1281 & $0.45^{* * *}$ & 666 & $0.52^{* * *}$ \\
\hline Severity of MJS & 1281 & $0.45^{* * *}$ & 666 & $0.52^{* * *}$ & - & - & - & - \\
\hline Severity of Worst Tiredness & 1281 & $0.32^{* * *}$ & 666 & $0.38^{* * *}$ & 1281 & $0.79^{* * *}$ & 666 & $0.77^{* * *}$ \\
\hline Severity of Worst Joint Pain & 1281 & $0.41^{* * *}$ & 666 & $0.47^{* * *}$ & 1281 & $0.89^{* * *}$ & 666 & $0.89^{* * *}$ \\
\hline \multicolumn{9}{|l|}{ SF-36 } \\
\hline Role Physical Domain & 1233 & $-0.23^{* * *}$ & 632 & $-0.29 * * *$ & 1233 & $-0.47^{* * *}$ & 632 & $-0.45^{* * *}$ \\
\hline Bodily Pain Domain & 1233 & $-0.31^{* * *}$ & 632 & $-0.36^{* * *}$ & 1233 & $-0.59 * * *$ & 632 & $-0.55^{* * *}$ \\
\hline General Health Domain & 1233 & $-0.15^{* * *}$ & 632 & $-0.19 * * *$ & 1233 & $-0.36^{* * *}$ & 632 & $-0.30^{* * *}$ \\
\hline Social Functioning Domain & 1233 & $-0.18^{* * *}$ & 632 & $-0.30^{* * *}$ & 1233 & $-0.43^{* * *}$ & 632 & $-0.46^{* * *}$ \\
\hline Vitality Domain & 1233 & $-0.19^{* * *}$ & 632 & $-0.29 * * *$ & 1233 & $-0.41^{* * *}$ & 632 & $-0.39 * * *$ \\
\hline Role Emotional Domain & 1233 & $-0.18^{* * *}$ & 632 & $-0.23^{* * *}$ & 1233 & $-0.39 * * *$ & 632 & $-0.33^{* * *}$ \\
\hline Mental Health Domain & 1233 & $-0.16^{* * *}$ & 632 & $-0.21^{* * *}$ & 1233 & $-0.33^{* * *}$ & 632 & $-0.30^{* * *}$ \\
\hline Physical Functioning Domain & 1233 & $-0.22^{* * *}$ & 632 & $-0.32^{* * *}$ & 1233 & $-0.49^{* * *}$ & 632 & $-0.46^{* * *}$ \\
\hline Mental Component Score & 1233 & $-0.14^{* * *}$ & 632 & $-0.21^{* * *}$ & 1233 & $-0.31^{* * *}$ & 632 & $-0.29^{* * *}$ \\
\hline Physical Component Score & 1233 & $-0.25^{* * *}$ & 632 & $-0.31^{* * *}$ & 1233 & $-0.52^{* * *}$ & 632 & $-0.48^{* * *}$ \\
\hline HAQ-DI Total Score & 1233 & $0.23^{* * *}$ & 632 & $0.31^{* * *}$ & 1233 & $0.55^{* * *}$ & 632 & $0.51^{* * *}$ \\
\hline
\end{tabular}


Table 3 Pearson Correlations between Duration of MJS, Severity of MJS, Severity of Worst Tiredness, and Other Instruments in RABEAM and RA-BUILD (Continued)

\begin{tabular}{|c|c|c|c|c|c|c|c|c|}
\hline \multirow[t]{3}{*}{ Instruments } & \multicolumn{4}{|c|}{ Duration of MJS } & \multicolumn{4}{|c|}{ Severity of MJS } \\
\hline & \multicolumn{2}{|c|}{ RA-BEAM } & \multicolumn{2}{|c|}{ RA-BUILD } & \multicolumn{2}{|c|}{ RA-BEAM } & \multicolumn{2}{|c|}{ RA-BUILD } \\
\hline & Number & $r$ & Number & $r$ & Number & $r$ & Number & $r$ \\
\hline QIDS-SR 16 Total Score & 1231 & $0.20^{* * *}$ & 632 & $0.24^{* * *}$ & 1231 & $0.34^{* * *}$ & 632 & $0.31^{* * *}$ \\
\hline Patient's Assessment of Pain & 1233 & $0.38^{* * *}$ & 632 & $0.39^{* * *}$ & 1233 & $0.78^{* * *}$ & 632 & $0.72^{* * *}$ \\
\hline PtGA & 1233 & $0.36^{* * *}$ & 632 & $0.37^{* * *}$ & 1233 & $0.74^{* * *}$ & 632 & $0.67^{* * *}$ \\
\hline Tender Joint Count 28 & 1231 & $0.16^{* * *}$ & 630 & $0.31^{* * *}$ & 1231 & $0.39^{* * *}$ & 630 & $0.44^{* * *}$ \\
\hline Swollen Joint Count 28 & 1231 & $0.16^{* * *}$ & 630 & $0.22^{* * *}$ & 1231 & $0.28^{* * *}$ & 630 & $0.31^{* * *}$ \\
\hline PhGA & 1224 & $0.21^{* * *}$ & 627 & $0.29 * * *$ & 1224 & $0.41^{* * *}$ & 627 & $0.44^{* * *}$ \\
\hline hsCRP & 1220 & $0.26^{* * *}$ & 623 & $0.37^{* * *}$ & 1220 & $0.57^{* * *}$ & 623 & $0.57^{* * *}$ \\
\hline
\end{tabular}

Abbreviations: HAQ-DI Health Assessment Questionnaire-Disability Index, $h s C R P$ high-sensitivity C-reactive protein, MJS morning joint stiffness, $n$ number of patients, PtGA Patient's Global Assessment of Disease Activity, PhGA Physician's Global Assessment of Disease Activity, QIDS-SR ${ }_{16}$ Quick Inventory of Depressive Symptomatology Self-Rated-16, SF-36 36-Item Short Form Health Survey version 2 with Acute recall

Pearson correlation coefficients with significance using $p$-values denoted as: ${ }^{*} \leq 0.05,{ }^{* *} \leq 0.01,{ }^{* *} \leq 0.001$

${ }^{\mathrm{a}}$ Daily average of 7 days preceding visit that contained at least 4 measurements [29]

Pain (RA-BEAM $r=0.79$ and 0.89 ; RA-BUILD $r=0.75$ and 0.89 ), patient's assessment of pain (RA-BEAM $r$ $=0.59$ and 0.78 ; RA-BUILD $r=0.54$ and 0.72$)$, and Severity of Worst Tiredness (RA-BEAM $r=0.66$ and 0.79; RA-BUILD $r=0.52$ and 0.77).

In contrast, smaller correlations between Duration and Severity of MJS with SF-36 MCS, SF-36 Role Emotional, and the QIDS-SR 16 at Day $1(r=-0.07$ to 0.34$)$ and Week $12(r=-0.39$ to 0.34$)$ were observed, indicating that the SF-36 and QIDS-SR 16 assessments measure more distally related constructs.

These findings demonstrate adequate convergent and discriminant validity, as well as the uniqueness of Duration of MJS and Severity of MJS compared to other PROs and clinical measures of RA in patients with moderately to severely active RA.

\section{Known-groups validity}

Due to small sample sizes in the lower DAS28-ESR subgroups at Day 1 (i.e., $<5 \%$ of the sample in each score category) in RA-BEAM and RA-BUILD, patients were categorized into only 2 subgroups: $\leq 5.1$ and $>5.1$ (Table 4 ). At Day 1 in both studies, patients with higher DAS28-ESR scores reported a significantly longer Duration of MJS measured in minutes (RA-BEAM: $p=0.028$; RA-BUILD: $p=0.033)$ and significantly greater Severity of MJS (RA-BEAM: $p=0.001$; RA-BUILD: $p=0.001$ ) than those patients with lower DAS28-ESR scores. Similar findings were evident for both studies at Week 4 .

Similar to the approach taken for the DAS28-ESR analyses, due to small sample sizes in the lower CDAI subgroups, patients were categorized into 2 subgroups based on the CDAI at Day 1 ( 0.0 to $\leq 22.0$ and $>22.0$ to $\leq 76.0)$ and 3 subgroups at Week $4(0.0$ to $\leq 10.0,>10.0$ to $\leq 22.0$, and $>22.0$ to $\leq 76.0$ ). At Day 1 , patients with higher CDAI scores experienced a significantly longer
Duration of MJS in RA-BUILD (median $=60.0$ vs. 120.0, $p=0.016)$ and greater Severity of MJS in both RABEAM and RA-BUILD (RA-BEAM: mean $=4.7$ vs. 5.9 , $p=0.001$; RA-BUILD: 3.7 vs. $5.9, p=0.001)$ than those patients with a lower CDAI score (Table 4). However, at Week 4, the Duration of MJS was significantly longer and the Severity of MJS was significantly greater in patients with higher CDAI scores than in patients with low CDAI scores in both studies (all $p=0.001$ ) (Table 5). All values increased linearly with higher CDAI scores, where all post-hoc comparisons using Scheffé adjustment were statistically significant $(p=0.001)$, except for the comparison of median Duration of MJS values between CDAI categories of 0.0 to $\leq 10.0$ versus $>10.0$ to $\leq 22.0$ in RA-BUILD. These findings indicate that the Duration of MJS and Severity of MJS PROs are able to distinguish between known groups based on disease severity.

\section{Responsiveness}

As hypothesized, the responsiveness of the Duration of MJS and Severity of MJS PROs was supported by large and statistically significant differences in median and mean change in Duration of MJS and Severity of MJS, respectively, from Day 1 to Week 12 between patients defined as responders or nonresponders on the basis of the ACR20 at Week 12 (Table 6). For example, for Duration of MJS in RA-BEAM, median change from Day 1 to Week 12 was -35.1 for responders in comparison to -7.0 for nonresponders $(p=0.001)$. Similarly, for Severity of MJS in RA-BEAM, mean change was -3.2 compared to -1.1 for responders and nonresponders, respectively $(p=0.001)$.

Comparable findings were seen when using DAS28hsCRP as an anchor, as pairwise comparisons assessing significant differences in median and mean change between DAS28-hsCRP groups of $<2.6$ versus $\geq 3.2 \quad(p=0.001$ for all comparisons), and $\geq 2.6$ and $<3.2$ versus $\geq 3.2$ 
Table 4 Known-Groups Validity of Duration of MJS and Severity of MJS Using DAS28-ESR Subgroups at Day 1 and Week 4, and CDAI Subgroups at Day 1

\begin{tabular}{|c|c|c|c|}
\hline \multirow[t]{2}{*}{ PRO/Study } & \multicolumn{2}{|c|}{ DAS28-ESR Category } & \multirow[t]{2}{*}{$p$-value, ES } \\
\hline & $\begin{array}{l}\leq 5.1 \\
\text { N, Mean (SD), Median }\end{array}$ & $\begin{array}{l}>5.1 \\
\text { N, Mean (SD), Median }\end{array}$ & \\
\hline \multicolumn{4}{|c|}{ Duration of MJS Day 1} \\
\hline RA-BEAM & $39,120.5(190.8), 60.0$ & 496, $155.3(180.1), 90.0$ & $0.028^{\mathrm{a}}, 0.19$ \\
\hline RA-BUILD & $36,132.8(191.5), 60.0$ & $273,164.9(173.1), 120$ & $0.033^{\mathrm{a}}, 0.18$ \\
\hline \multicolumn{4}{|c|}{ Duration of MJS Week 4} \\
\hline RA-BEAM & $619,76.1$ (108.6), 37.3 & $587,133.6(153.5), 73.6$ & $0.001^{a}, 0.43$ \\
\hline RA-BUILD & $327,77.6(112.0), 40.0$ & 286, $151.1(158.3), 94.6$ & $0.001^{\mathrm{a}}, 0.54$ \\
\hline \multicolumn{4}{|c|}{ Severity of MJS at Day 1} \\
\hline RA-BEAM & $39,4.4(2.7)$ & $496,5.9(2.1)$ & $0.001^{b}, 0.70$ \\
\hline RA-BUILD & $36,4.0,(1.7)$ & $274,5.9(2.0)$ & $0.001^{b}, 0.97$ \\
\hline \multicolumn{4}{|c|}{ Severity of MJS at Week 4} \\
\hline RA-BEAM & $619,3.0(1.9)$ & $587,5.1(2.1)$ & $0.001^{b}, 1.05$ \\
\hline \multirow[t]{3}{*}{ RA-BUILD } & $327,3.1(1.9)$ & $286,5.3(2.0)$ & $0.001^{b}, 1.13$ \\
\hline & \multicolumn{2}{|c|}{ CDAI Category } & $p$-value, ES \\
\hline & $\begin{array}{l}0.0 \text { to } \leq 22.0 \\
N, \text { Mean (SD), Median }\end{array}$ & $\begin{array}{l}>22.0 \text { to } \leq 76.0 \\
\text { N, Mean (SD), Median }\end{array}$ & \\
\hline \multicolumn{4}{|c|}{ Duration of MJS Day 1} \\
\hline RA-BEAM & 36, 144.1 (198.3), 90.0 & $497,153.9(180.0), 90.0$ & $0.305^{\mathrm{a}}, 0.05$ \\
\hline RA-BUILD & $26,111.9(162.7), 60.0$ & $278,162.3(170.6), 120$ & $0.016^{\mathrm{a}}, 0.30$ \\
\hline \multicolumn{4}{|c|}{ Severity of MJS at Day 1} \\
\hline RA-BEAM & $36,4.7(2.4)$ & $497,5.9(2.1)$ & $0.001^{b}, 0.57$ \\
\hline RA-BUILD & $26,3.7(1.6)$ & $278,5.9(2.1)$ & $0.001^{b}, 1.07$ \\
\hline
\end{tabular}

Abbreviations: CDAI Clinical Disease Activity Index, DAS28 Disease Activity Score modified to include the 28 diarthrodial joint count, ESR erythrocyte sedimentation rate, MJS morning joint stiffness, $S D$ standard deviation, ES effect size

${ }^{a} p$-value based on a comparison of median values using the Kruskal-Wallis test

${ }^{\mathrm{b}} p$-value based on a comparison of mean values using ANOVA

Table 5 Known-Groups Validity of Duration of MJS and Severity of MJS Using CDAI Subgroups at Week 4

\begin{tabular}{|c|c|c|c|c|c|}
\hline \multirow[t]{2}{*}{ PRO/Study } & \multicolumn{3}{|l|}{ CDAI Category } & \multirow[t]{2}{*}{$P$-value } & \multirow[t]{2}{*}{ Comparisons, ES } \\
\hline & $\begin{array}{l}0.0 \text { to } \leq 10.0 \\
N \text {, Mean (SD), Median }\end{array}$ & $\begin{array}{l}>10.0 \text { to } \leq 22.0 \\
\mathrm{~N}, \text { Mean (SD), Median }\end{array}$ & $\begin{array}{l}>22.0 \text { to } \leq 76.0 \\
\text { N, Mean (SD), Median }\end{array}$ & & \\
\hline \multicolumn{6}{|c|}{ Duration of MJS Week at Week 4} \\
\hline RA-BEAM & 220, 58.3 (95.0), 20.4 & 426, $90.1(121.8), 47.1$ & $567,133.0(152.8), 75.7$ & $0.001^{a}$ & a: $0.001,0.28 ; b: 0.001,0.54 ; c: 0.001,0.31$ \\
\hline RA-BUILD & $134,70.5(107.1), 31.4$ & $209,88.1(123.3), 45.7$ & $275,152.9(158.9), 95.0$ & $0.001^{\mathrm{a}}$ & a: $0.146,0.15 ;$ b: $0.001,0.57 ;$ c: $0.001,0.45$ \\
\hline \multicolumn{6}{|c|}{ Severity of MJS at Week 4} \\
\hline RA-BEAM & $220,2.3(1.7)$ & $426,3.6(2.0)$ & $567,5.0(2.1)$ & $0.001^{\mathrm{b}}$ & a: $0.001,0.68 ;$ b: $0.001,1.35 ; c: 0.001,0.68$ \\
\hline RA-BUILD & $134,2.5(1.8)$ & $209,3.6(1.8)$ & $275,5.4(2.0)$ & $0.001^{b}$ & a: $0.001,0.61 ;$ b: $0.001,1.50 ; c: 0.001,0.94$ \\
\hline
\end{tabular}

Abbreviations: CDAI Clinical Disease Activity Index, MJS morning joint stiffness, SD standard deviation, ES effect size

${ }^{a} p$-value based on a comparison of median values using the Kruskal-Wallis test

${ }^{\mathrm{b}} p$-value based on a comparison of mean values using analysis of variance

${ }^{c}$ Note for multiple comparison using Scheffé adjustment: a: 0.0 to $\leq 10.0$ versus $>10.0$ to $\leq 22.0$; b: 0.0 to $\leq 10.0$ versus $>22.0$ to $\leq 76.0$; c: $>10.0$ to $\leq 22.0$ versus $>22.0$

to $\leq 76.0$. Effect size estimates were calculated on the unadjusted group scores 
Table 6 Change from Day 1 to Week 12 among ACR20 and DAS28-hsCRP Groups

\begin{tabular}{|c|c|c|c|c|c|c|c|}
\hline \multirow{2}{*}{$\begin{array}{l}\text { PRO/Study/ } \\
\text { Statistics }\end{array}$} & \multicolumn{2}{|c|}{ ACR20 Category at Week 12} & \multirow[t]{2}{*}{$P$-value, ES } & \multicolumn{3}{|c|}{ DAS28-hsCRP Groups at Week $12^{\mathrm{b}}$} & \multirow{2}{*}{$\begin{array}{l}\text { Comparisons, } \\
E^{c}\end{array}$} \\
\hline & Responder $^{a}$ & Nonresponder $^{a}$ & & $\begin{array}{l}\text { DAS28-hsCRP } \\
<2.6\end{array}$ & $\begin{array}{l}2.6 \leq \text { DAS28-hsCRP } \\
<3.2\end{array}$ & $\begin{array}{l}\text { DAS28-hsCRP } \\
\geq 3.2\end{array}$ & \\
\hline \multicolumn{8}{|l|}{ Duration of MJS } \\
\hline \multicolumn{8}{|l|}{ RA-BEAM } \\
\hline N, Mean (SD) & $326,-84.2(167.5)$ & $211,-17.2(166.4)$ & & $88,-92.4(185.0)$ & $79,-84.0(170.1)$ & $368,-44.2(165.4)$ & \\
\hline \multirow[t]{3}{*}{ Median $^{d}$} & -35.1 & -7.0 & $0.001,-0.40$ & -55.0 & -30.0 & -12.6 & a: $0.809,-0.05$ \\
\hline & & & & & & & b: $0.001,-0.28$ \\
\hline & & & & & & & c: $0.001,-0.24$ \\
\hline \multicolumn{8}{|l|}{ RA-BUILD } \\
\hline N, Mean (SD) & $174,-70.1(139.3)$ & $137,-19.6(196.8)$ & & $61,-87.9(133.7)$ & $33,-65.7(96.7)$ & $214,-34.1(185.0)$ & \\
\hline \multirow[t]{3}{*}{ Median $^{d}$} & -42.9 & -9.1 & $0.001,-0.30$ & -50.7 & -40.0 & -26.9 & a: $0.221,-0.18$ \\
\hline & & & & & & & b: $0.001,-0.31$ \\
\hline & & & & & & & c: $0.022,-0.18$ \\
\hline \multicolumn{8}{|l|}{ Severity of MJS } \\
\hline \multicolumn{8}{|l|}{ RA-BEAM } \\
\hline \multirow[t]{3}{*}{ N, Mean (SD) } & $326,-3.2(2.3)$ & $211,-1.1(2.2)$ & $0.001,-0.90$ & $88,-3.7(2.3)$ & $79,-3.1(2.3)$ & $368,-1.9(2.4)$ & a: $0.083,-0.25$ \\
\hline & & & & & & & b: $0.001,-0.76$ \\
\hline & & & & & & & c: $0.001,-0.52$ \\
\hline \multicolumn{8}{|l|}{ RA-BUILD } \\
\hline \multirow[t]{3}{*}{ N, Mean (SD) } & $175,-2.9(2.3)$ & $137,-1.0(2.1)$ & $0.001,-0.85$ & $61,-3.1(2.5)$ & $33,-2.6(2.6)$ & $215,-1.8(2.2)$ & a: $0.264,-0.21$ \\
\hline & & & & & & & b: $0.001,-0.59$ \\
\hline & & & & & & & c: $0.002,-0.35$ \\
\hline
\end{tabular}

Abbreviations: ACR20 20\% improvement in American College of Rheumatology criteria, DAS28 Disease Activity Score modified to include the 28 diarthrodial joint count, $h S C R P$ high-sensitivity C-reactive protein, MJS morning joint stiffness, SD standard deviation, ES effect size

${ }^{a}$ Responder: Achievement of ACR20 criteria at Week 12; Nonresponder: Failure to achieve ACR20 criteria at Week 12

${ }^{\mathrm{b}}$ Missing DAS28-hsCRP at Week 12 was imputed using modified baseline observation carried forward

'Note for multiple comparison: a: $<2.6$ vs. $\geq 2.6$ and $<3.2$; b: $<2.6$ vs. $\geq 3.2$; c: $\geq 2.6$ and $<3.2$ vs. $\geq 3.2$

${ }^{d}$ Daily average of 7 days preceding visit that contained at least 4 measurements

were statistically significant $(p<0.01$ for all comparisons) (Table 6). However, the change scores comparisons between $<2.6$ versus $\geq 2.6$ and $<3.2$ were not statistically significant for Duration of MJS (RA-BEAM $p=0.809$; RA-BUILD $p=0.221$ ) or Severity of MJS (RA-BEAM $p=0.083$; RA-BUILD $p=0.264$ ).

\section{Discussion}

The psychometric properties, including reliability, validity, and responsiveness, of Duration of MJS and Severity of MJS PROs were supported by the results from RABEAM and RA-BUILD. Specifically, test-retest reliability analyses revealed high levels of agreement in Duration of MJS and Severity of MJS values among patients considered stable across two assessment periods. In support of construct validity, a priori hypotheses of the relationships between Duration of MJS and Severity of MJS with other related PROs and clinical outcome measures were supported at Day 1 and Week 12 assessment periods. Known-groups validity was similarly supported as median and mean values of Duration of MJS and
Severity of MJS were significantly different based on known-groups using the DAS28-ESR and CDAI. Finally, both measures were responsive to change from Day 1 to Week 12 when defining responders using the ACR20. Sensitivity to change was also supported when using the DAS28-hsCRP as an anchor, except for comparisons between the lowest disease activity groups.

However, although these symptoms have been demonstrated to be important to patients with RA, an assessment of these symptoms was excluded from the the core set of disease activity measures. Specifically, rheumatologists have frequently used morning stiffness as an indicator that patient medication changes are needed [25]. However, in 1993, the core set of disease activity measures for use in RA clinical trials was revised. Studies with therapies available at the time (e.g., csDMARDs, auranofin, nonsteroidal anti-inflammatory drugs [NSAIDs]) suggested that MJS was not sensitive to change with treatment compared to control, and this lack of ability to discriminate treatment from control contributed to this symptom's exclusion from the core set of measures [26]. 
Subsequent to its removal from the ACR core set in 1993, morning stiffness has been found to be an important determining clinical status indicator driving changes to DMARD therapy [27]. Additional work by Boers et al. [28] further demonstrated that both duration and severity of morning stiffness are related to other measures of disease activity. The findings from the present study provide support that both the Duration of MJS and Severity of MJS PROs are not only associated with other measures of disease activity in RA but rather are measures that assess distinct symptom experiences by patients. Furthermore, these measures are sensitive to detect change over time in adults with moderately to severely active RA. However, future research is needed in order to determine the relationship of these instruments to patient outcomes over a long term period, and the usability of such instruments in a clinical setting. Given the advent of electronic PRO diaries, these instruments could be used in a clinical setting and collected daily, thus enhancing the dialogue between patients and care providers. Further, using applications on mobile phones, these instruments can be easily completed by patients to track and collect their daily symptoms.

Despite the strong findings related to the reliability, validity, and responsiveness of the Duration of MJS and Severity of MJS PROs, a key limitation to this investigation is the missing data at the Day 1 assessment. As noted above, the missing data were due to a variety of reasons (e.g., device alarm not sounding until the following day). However, sensitivity analyses were conducted after imputing missing Day 1 Duration of MJS and Severity of MJS PRO scores, and results for the evaluations of reliability, validity, and responsiveness of these two PROs remained the same. In addition, the evaluation of responsiveness was limited as the studies' baseline assessment period consisted of only data at Day 1 rather than a full 7 days of data as was available at Week 12. Finally, all analyses were pooled across countries due to small sample sizes $(<5$ participants) within certain participating countries. Thus, future research is needed to assess the psychometric properties of both instruments within each country.

\section{Conclusion}

The findings from the current study demonstrate that the Duration of MJS and Severity of MJS items are unique PROs fit for purpose of measuring two key symptoms of MJS in adult patients with moderately to severely active RA in clinical trials.

\section{Additional file}

Additional file 1: Table S1. Descriptive Statistics for Duration of MJS and Severity of MJS at Day 1. Table S2 Reasons for Missing Diary Data at Day 1 for RA-BEAM and RA-BUILD. (DOCX 24 kb)

\section{Abbreviations}

ACR: American College of Rheumatology; ACR20: 20\% improvement in American College of Rheumatology criteria; ANCOVA: Analysis of covariance; ANOVA: Analysis of variance; CDAl: Clinical Disease Activity Index; CsDMARD: Conventional synthetic DMARD; DAS28: Disease Activity Score modified to include the 28 diarthroidal joint count; DMARD: Diseasemodifying antirheumatic drug; DSM-IV: American Psychiatric Association's Diagnostic and Statistical Manual of Mental Disorders, 4th Edition; ESR: Erythrocyte sedimentation rate; HAQ-DI: Health Assessment Questionnaire-Disability Index; HRQOL: Health-related quality of life; hsCRP: High-sensitivity C-reactive protein; ICC: Intraclass correlation coefficient; IL-6: Interleukin-6; MCS: Mental Component Score; MJS: Morning Joint Stiffness; NSAID: Nonsteroidal anti-inflammatory drug; PCS: Physical Component Score; PhGA: Physician's Global Assessment of Disease Activity; PRO: Patient-reported outcome; PtGA: Patient's Global Assessment of Disease Activity; QIDS-SR ${ }_{16}$ : Quick Inventory of Depressive Symptomatology SelfRated-16; RA: Rheumatoid arthritis; SDAl: Simplified Disease Activity Index; SF-36: Medical Outcomes Study 36-Item Short Form Health Survey Version 2 Acute; SJC: Swollen joint count; TJC: Tender joint count; TNF: Tumor necrosis factor; VAS: Visual analogue scale

\section{Acknowledgements}

Not applicable.

\section{Funding}

This study was funded by Eli Lilly and Company and Incyte.

\section{Availability of data and materials}

Lilly provides access to the individual patient data from studies on approved medicines and indications as defined by the sponsor specific information on ClinicalStudyDataRequest.com.

This access is provided in a timely fashion after the primary publication is accepted. Researchers need to have an approved research proposal submitted through clinicalstudydatarequest.com. Access to the data will be provided in a secure data sharing environment after signing a data sharing agreement.

\section{Authors' contributions}

All authors contributed substantially to the design of the study, the interpretation of the results, and the editing of the manuscript. All authors read and approved the final manuscript.

\section{Ethics approval and consent to participate}

The study was approved by the Copernicus Group IRB in Research Triangle Park, NC (IRB Tracking \#: PAR1-09-143). Ethics approval was also obtained for all 41 sites. All patients provided written informed consent.

Consent for publication

Not applicable.

\section{Competing interests}

EDB and KWW were employees of Evidera, and received financial support from Eli Lilly in connection with the development of the manuscript. AMD, $\mathrm{CL}, \mathrm{CLG}, \mathrm{TR}$, and RH are all full-time employees of Eli Lilly and Company. TR is a minor share holder of Eli Lilly and Company.

\section{Publisher's Note}

Springer Nature remains neutral with regard to jurisdictional claims in published maps and institutional affiliations.

\section{Author details \\ ${ }^{1}$ Evidera, Inc, 1417 4th Avenue, Suite 510, Seattle, WA 98101, USA. ${ }^{2}$ Eli Lilly and Company, Indianapolis, IN, USA. ${ }^{3}$ Evidera, Inc, Bethesda, MD, USA.}

Received: 21 April 2017 Accepted: 27 November 2017

Published online: 06 December 2017

References

1. Straub RH, Cutolo M. Circadian rhythms in rheumatoid arthritis: implications for pathophysiology and therapeutic management. Arthritis Rheum. 2007;56:399-408. 
2. Cutolo M, Otsa K, Aakre O, Sulli A. Nocturnal hormones and clinical rhythms in rheumatoid arthritis. Ann N Y Acad Sci. 2005;1051:372-81.

3. Gibbs JE, Ray DW. The role of the circadian clock in rheumatoid arthritis. Arthritis Res Ther. 2013;15:205.

4. Westhoff G, Buttgereit F, Gromnica-Ihle E, Zink A. Morning stiffness and its influence on early retirement in patients with recent onset rheumatoid arthritis. Rheumatology (Oxford). 2008;47:980-4.

5. Mattila K, Buttgereit F, Tuominen R. Impact of morning stiffness on working behaviour and performance in people with rheumatoid arthritis. Rheumatol Int. 2014;34:1751-8.

6. Sierakowski S, Cutolo M. Morning symptoms in rheumatoid arthritis: a defining characteristic and marker of active disease. Scand J Rheumatol Suppl. 2011;125:1-5.

7. Sokka T. Morning stiffness and other patient-reported outcomes of rheumatoid arthritis in clinical practice. Scand J Rheumatol Suppl. 2011;125:23-7.

8. DeLozier AM, Gaich CL, Vernon MK, von Maltzahn R. Content validity evaluation of a new diary developed to evaluate symptoms important to patients with moderate-to-severe rheumatoid arthritis. Philadelphia: ISPOR 20th Annual International Meeting; May 16-20; 2015

9. Taylor PC, Keystone EC, van der Heijde D, Weinblatt ME, Del Carmen ML, Reyes Gonzaga J, Yakushin S, Ishii T, Emoto K, Beattie S, et al. Baricitinib versus placebo or Adalimumab in rheumatoid arthritis. $\mathrm{N}$ Engl J Med. 2017;376:652-62

10. Dougados M, van der Heijde D, Chen YC, Greenwald M, Drescher E, Liu J, Beattie S, Witt S, de la Torre I, Gaich C, et al. Baricitinib in patients with inadequate response or intolerance to conventional synthetic DMARDs: results from the RA-BUILD study. Ann Rheum Dis. 2017;76:88-95.

11. Ware JE Jr, Sherbourne CD. The MOS 36-item short-form health survey (SF36). I. Conceptual framework and item selection. Med Care. 1992:30:473-83.

12. McHorney CA, Ware JE Jr, Lu JF, Sherbourne CD. The MOS 36-item shortform health survey (SF-36): III. Tests of data quality, scaling assumptions, and reliability across diverse patient groups. Med Care. 1994;32:40-66.

13. Tsai C, Bayliss MS, Ware JE. SF-36 health survey annotated bibliography: second edition (1988-1996). Boston: Health Assessment Lab, New England Medical Center; 1997.

14. Fries JF, Spitz PW, Young DY. The dimensions of health outcomes: the health assessment questionnaire, disability and pain scales. J Rheumatol. 1982:9:789-93.

15. Cuperus N, Mahler EA, Vliet Vlieland TP, Hoogeboom TJ, van den Ende CH. Measurement properties of the health assessment questionnaire disability index for generalized osteoarthritis. Rheumatology (Oxford). 2015;54:821-6.

16. American Psychiatric Association. Diagnostic and statistical manual of mental disorders (DSM-IV). 4th ed. Washington: American Psychiatric Association; 1994.

17. Rush AJ, Trivedi MH, Ibrahim HM, Carmody TJ, Arnow B, Klein DN, Markowitz JC, Ninan PT, Kornstein S, Manber R, et al. The 16-item quick inventory of depressive Symptomatology (QIDS), clinician rating (QIDS-C), and self-report (QIDS-SR): a psychometric evaluation in patients with chronic major depression. Biol Psychiatry. 2003;54:573-83.

18. Aletaha D, Smolen J. The simplified disease activity index (SDAI) and the clinical disease activity index (CDAl): a review of their usefulness and validity in rheumatoid arthritis. Clin Exp Rheumatol. 2005;23:S100-8.

19. Felson DT, Smolen JS, Wells G, Zhang B, van Tuyl LH, Funovits J, Aletaha D, Allaart CF, Bathon J, Bombardieri S, et al. American College of Rheumatology/ European league against rheumatism provisional definition of remission in rheumatoid arthritis for clinical trials. Ann Rheum Dis. 2011;70:404-13.

20. Fransen J, Stucki G, van Riel PLCM. Rheumatoid arthritis measures: disease activity score (DAS), disease activity Score-28 (DAS28), rapid assessment of disease activity in rheumatology (RADAR), and rheumatoid arthritis disease activity index (RADAI). Arthritis Care Res. 2003;49:S214-24.

21. DeLoach LJ, Higgins MS, Caplan AB, Stiff JL. The visual analog scale in the immediate postoperative period: intrasubject variability and correlation with a numeric scale. Anesth Analg. 1998;86:102-6.

22. Nunnally JC, Bernstein IH. Psychometric theory. 3rd ed. New York: McGraw-Hill; 1994.

23. Cohen J. Statistical power analysis for the behavioral sciences. 2nd ed. Hillsdale: Lawrence Erlbaum Associates; 1988.

24. Koch GG, Tangen CM, Jung JW, Amara IA. Issues for covariance analysis of dichotomous and ordered categorical data from randomized clinical trials and non-parametric strategies for addressing them. Stat Med. 1998;17:1863-92.
25. Kirwan JR, Chaput de Saintonge DM, Joyce CR, Currey HL. Clinical judgment in rheumatoid arthritis. III. British rheumatologists' judgments of 'change in response to therapy'. Ann Rheum Dis. 1984;43:686-94.

26. Felson DT, Anderson JJ, Boers M, Bombardier C, Chernoff M, Fried B, Furst D, Goldsmith C, Kieszak S, Lightfoot R, et al. The American College of Rheumatology preliminary core set of disease activity measures for rheumatoid arthritis clinical trials. The committee on outcome measures in rheumatoid arthritis clinical trials. Arthritis Rheum. 1993;36:729-40.

27. Soubrier M, Zerkak D, Gossec L, Ayral X, Roux C, Dougados M. Which variables best predict change in rheumatoid arthritis therapy in daily clinical practice? J Rheumatol. 2006;33:1243-6.

28. Boers M, Buttgereit F, Saag K, Alten R, Grahn A, Storey D, Rice P, Kirwan J. What is the relationship between morning symptoms and measures of disease activity in patents with rheumatoid arthritis. Arthrit Care Res. 2015; 67:1202-9.

\section{Submit your next manuscript to BioMed Central and we will help you at every step:}

- We accept pre-submission inquiries

- Our selector tool helps you to find the most relevant journal

- We provide round the clock customer support

- Convenient online submission

- Thorough peer review

- Inclusion in PubMed and all major indexing services

- Maximum visibility for your research

Submit your manuscript at www.biomedcentral.com/submit 